\title{
The evaluation of unilateral closed-lip schizencephaly on complex MRI sequences
}

\author{
Emrah Doğan \\ Radiology, Faculty of Medicine, Muğla Sıtkı Koçman University, Mugla, Turkey
}

\begin{abstract}
Schizencephaly is one of the rare cortical malformations that classify in the group of neurological migration defects. The appearance of the anomaly is in the form of a cleft that passes through the brain parenchyma. In mild types, the cleft does not reach the lateral ventricle. Demonstrating the presence of polymicrogyria, optimal evaluation of the cortex and determining the cleft trace are essential in the radiological evaluation of schizencephaly. Because differential diagnosis should be made with heterotopias and focal cortical dysplasia. We present a case of a 21-yearold female patient with closed-lip schizencephaly accompanied by MRI findings. Polymicrogyria is best detected in sagittal T2 sequences, while the difference of white matter and grey matter are well observed in T1 and FLAIR sequences. The benefit of diffusion-weighted images in schizencephaly is unclear. The ADC values of the grey matter adjacent to the cleft are close to the lower limit of normal. Susceptibility weighted image (SWI) sequence has no place in the evaluation of schizencephaly. It can be beneficial for the exclusion of vascular malformations, haemorrhage and differential diagnosis.
\end{abstract}

Keywords: schizencephaly, closed-lip, MRI, MRI sequences

\section{INTRODUCTION}

Schizencephaly is a rare cortical malformation in the form of a cleft that seen throughout the brain parenchyma (1). The type extending to the lateral ventricle is called open-lip, while the type ending in the white matter is called closed-lip. $3 / 4$ of the cases are open-lip, and $1 / 4$ are closed-lip. Schizencephaly is rare, with an estimated incidence of approximately $1.48 / 100,000$ live births. In other words, the prevalence of closed-lip Schizencephaly is $1 / 400,000$ in the normal population. It is almost always sporadic, although few familial cases have been described. MRI is the most detailed radiological method used to evaluate schizencephaly (2). Herein, apart from T1W, T2W turbo spin-echo and FLAIR sequences, Diffusion-ADC, contrast and SWI (magnitude, filtered phase, SWI (combined post-processed magnitude and phase), SWI minimum intensity projection (MIP) views of schizencephaly were demonstrated. Our paper aims to demonstrate the radiological evaluation process and the use of sequences in closed-lip schizencephaly. We present a case of a 21-year-old female patient accompanied by MRI images. The consent form was signed by the patient.

\section{CASE PRESENTATION}

A 21-year-old female patient presented to the hospital with a one-week history of headache. The headache was localized to the occipital region and bilateral. There was a history of hypertension in the patient's past. There were no past remarkable medical history, no smoking history, or relevant family history. No additional feature was found in his neurological and systemic examination. MRI was requested. A closed-lip cleft appearance was 
observed. The cleft terminated in the form of the blind zone by widening. The cleft was located in the course of the left postcentral gyrus. In the T1-weighted sequence, the cortex band around the cleft was clearly visualized. The band of grey matter was thinner at the end of the cleft (Figure 1A). The cortical micro-irregularities of polymicrogyria were more prominent in the outer part of the cleft. Polymicrogyria was more clearly visualized in sagittal T2 weighted sequences (Figure 1B). In the FLAIR sequences, the MRI signal was suppressed in the cleft space. That was evidence that the fluid was cerebrospinal fluid (CSF). Grey matter and white matter difference were better evaluated in the FLAIR sequence compared to other sequences. The FLAIR signal of the cortex around the expanding blind zone was higher. The grey matter was also thinner in this area. That was the evidence of abnormal cortex tissue (Figure 1C). In contrast-enhanced images, emissary veins along the cleft were observed (Figure 1D).
No signal that creates susceptibility effect was detected in magnitude images, filtered-phase images, SWI (combined post-processed magnitude and phase) and minimum intensity projection (MIP) images. In magnitude images, it is not possible to detect the difference between grey and white matters. The cleft was hypointense in this sequence and similar to the CFS signal (Figure 2A). There was no remarkable finding in phaseimages and SWI since these sequences have a susceptibility to calcification or haemorrhage (Figure $2 \mathrm{~B}, \mathrm{C})$. The vascular structures were clearly visible on the MIP images. There were no remarkable findings except well-visualized emissary veins (Figure 2D).

There was no diffusion restriction in diffusion-weighted images. The ADC value was of $750-800 \times 10^{-6} \mathrm{~mm}^{2} / \mathrm{s}$. ADC values were at the lower limit of the normal grey matter scale (Figure 3 ). MRI findings were compatible with closedtype schizencephaly.

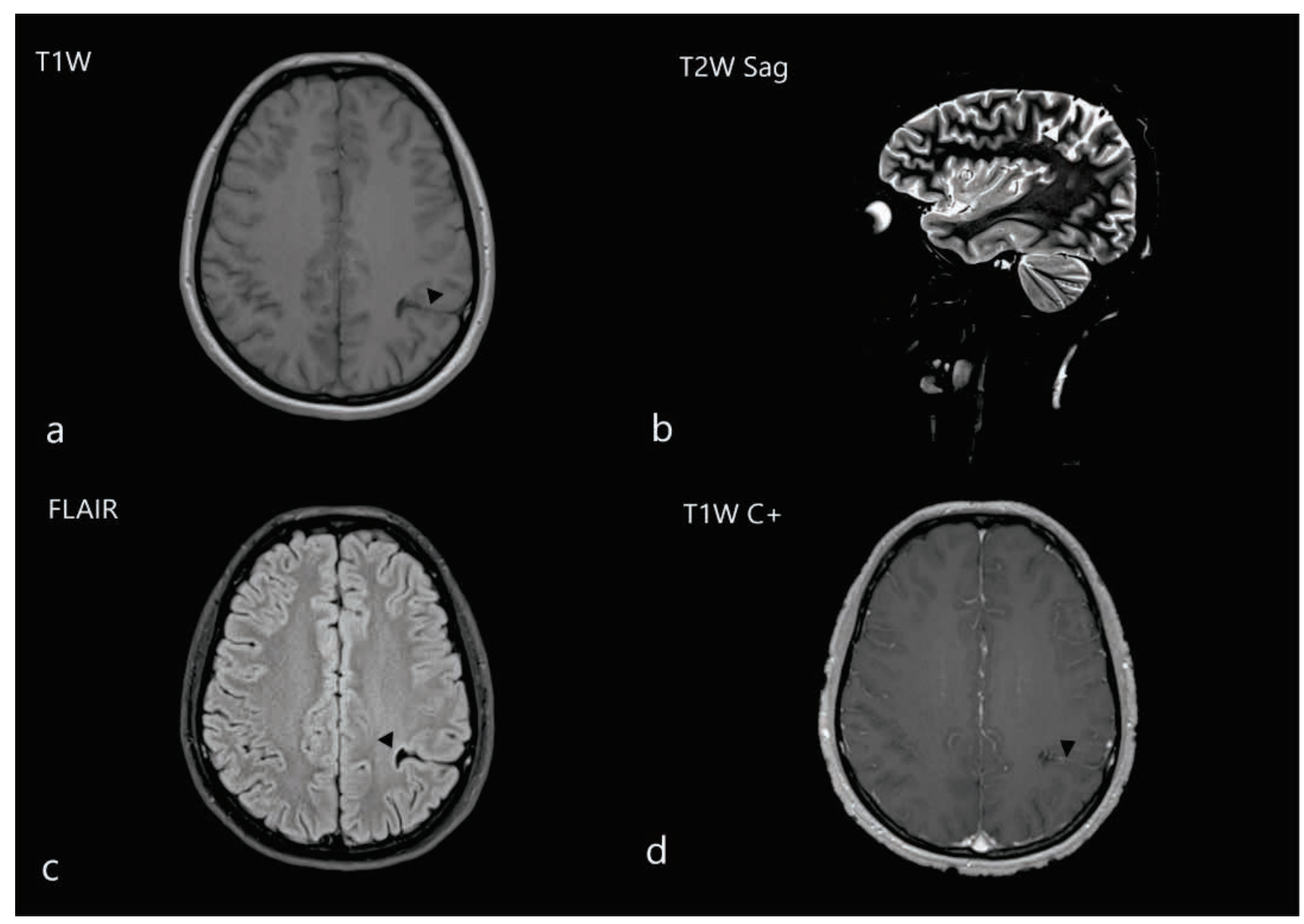

FIGURE 1. a. Axial T1 weighted MRI (arrowhead: The cleft of schizencephaly) B. Sagittal T2 weighted images (arrowhead: polymicrogyria) C. FLAIR sequence (arrowhead: cleft terminated with hyperintense cortex) D. Contrast enhanced MRI (arrowhead: emissary veins in the cleft) 


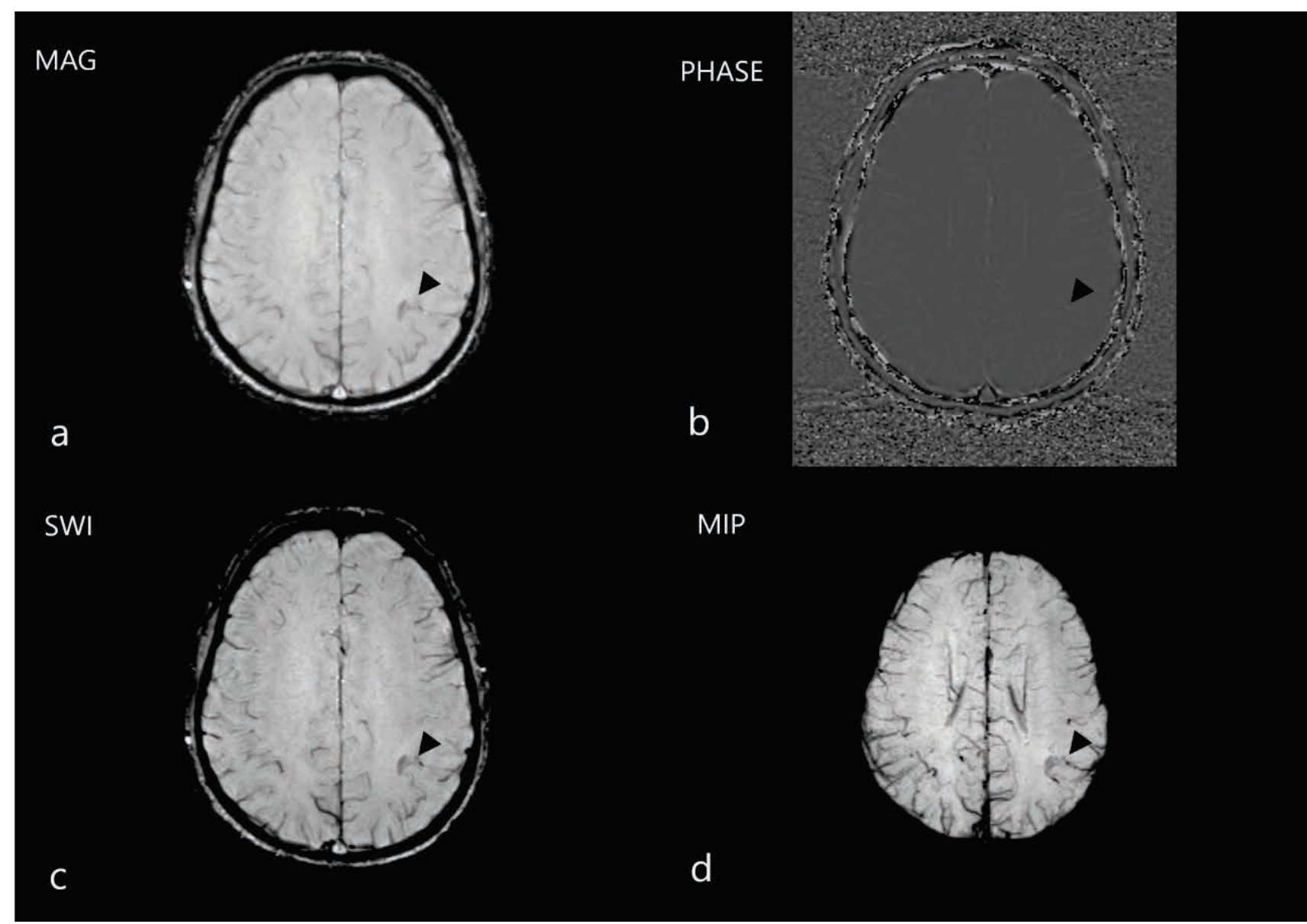

FIGURE 2. A. Magnitude images (arrowhead: The cleft is seen in fluid signal same to CFS) B. Phase image (arrowhead: The cleft line is tentative). C. The cleft is seen in SWI sequence but grey and white matter difference is not clear. D. Vascular structures are well defined in MIP images. Emissary veins are seen in this sequence

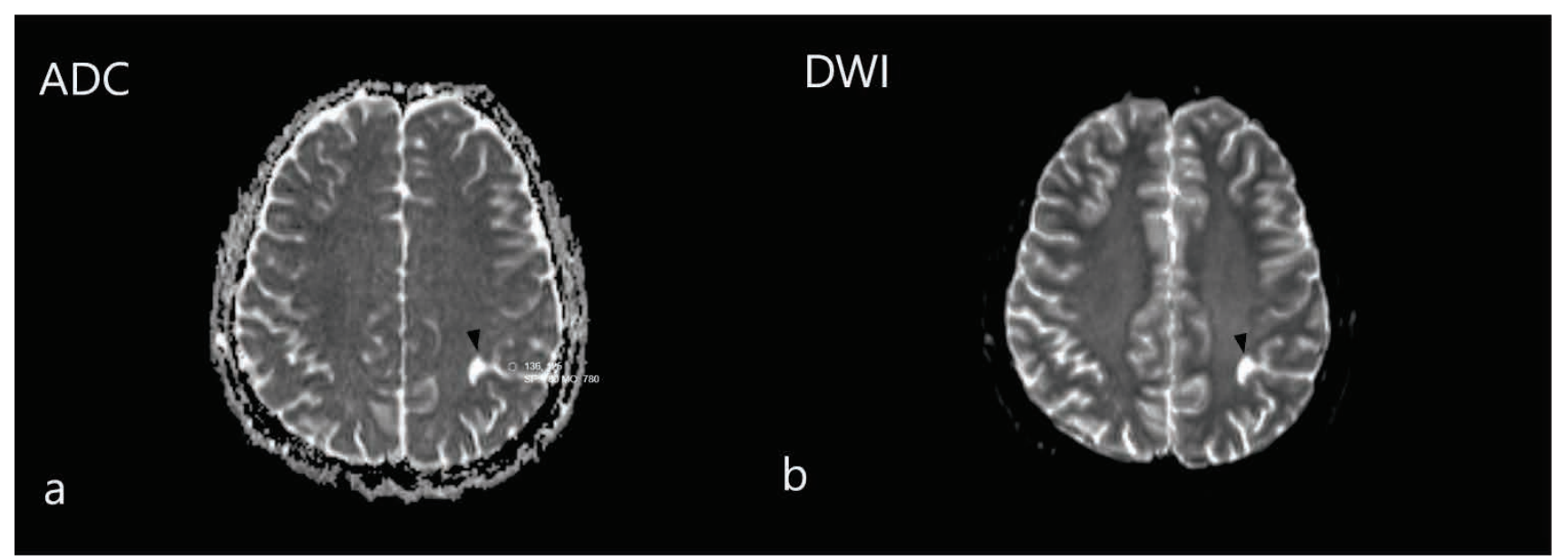

FIGURE 3. A. Hyper intense cleft bant is well visualized in ADC sequence. ADC values was at the lower limit of the normal grey matter scale $B$. The cleft was also hyperintense in DWI sequence due to CFS signal

\section{DISCUSSION}

Schizencephaly is a congenital brain malformation classified among neuronal migration defects together with lissencephaly, heterotopia and polymicrogyria. It is caused by a developmental defect that occurs between the intrauterine 2 nd and 5th weeks. Polymicrogyria accompanies schizencephaly along the cleft (3). Some authors do not use the term schizencephaly. They generally prefer to group these disorders under the term porencephaly. However, Schizencephaly is a true malformation. Porencephaly is the loss of brain tissue caused by an encephaloclastic event (e.g., ischemia) (2). 
Interestingly, schizencephaly is more common in abandoned or adopted children (3). Other possible etiological factors include exposure to teratogenic agents, prenatal viral infections, genetics and young maternal age (4). Some familial cases have been reported. Heterozygous germline mutations of the homeobox gene EMX2 are frequently encountered (5). There is no known gender preference.

The clinical features of schizencephaly patients can be classified according to whether the finding is unilateral or bilateral. Unilateral schizencephaly presents contralateral hemiparesis and asymmetrical muscle tone. Bilateral schizencephaly generally may manifest with seizures, developmental delay, quadriparesis, and severe mental disorders. The severity and extent of seizures depend on the anatomical extent of the involvement. General developmental delay and motor disorders are expected findings in bilateral Schizencephaly $(2,6)$. Schizencephaly is classified into three groups.

- Schizencephaly (type 1) - abnormal grey matter but no evidence of cleft on MRI

- Schizencephaly (type 2) - cleft is present, but abnormal lips are adjacent

- Schizencephaly (type 3) - cleft is present, but abnormal lips are not adjacent (7).

Our case is compatible with type 2 schizencephaly.

Neighbouring meninges accompany parenchymal pathology in the area of schizencephaly. A cleft extends to the pia mater from the ependymal surface of the brain. The two layers merge at the cleft. Often the cleft affects the posterior frontal or parietal lobes. Large clefts can affect a large area, including the temporal or occipital lobes. However, isolated involvement of these lobes is rare (8).

Schizencephaly is often associated with other cerebral anomalies: the most frequently associated anomalies are septo-optic dysplasia, grey matter heterotopia, absence of Septum pellucidum, corpus callosum dysgenesis (9).

MRI is the preferred imaging method in the evaluation of schizencephaly. It provides visualization of cortical dysplasia and heterotopic grey matter as well as identification of the pial-ependymal cleft. Each of the MR sequences used is effective in showing different characteristics of schizencephaly. The cortex detail of schizencephaly is clearly seen in the T1-weighted sequence. In
T1-weighted sequences, white matter and grey matter are in the intermediate signal. However, white matter is slightly more hyperintense than grey matter. In the T1-weighted sequence, the fluid filling the cleft is hypointense. In addition, the T1-weighted sequence is important in differential diagnosis. Bleeding is not an expected finding in schizencephaly. However, in the case of cortical laminar necrosis, if the sulcus enlargement occurs, this false appearance may be confused with the mild form schizencephaly. Herein, T1 hyperintensity of bleeding is helpful in the differential diagnosis (10).

T2 weighted sequences in the sagittal plan are used in the evaluation of polymicrogyria. Polymicrogyria cortex areas adjacent to hyperintense CSF can be evaluated in detail in the T2 sequence. Also, areas of porencephaly can be evaluated more clearly in the T2-weighted sequence.

FLAIR sequence is very valuable in evaluating schizencephaly. Focal cortical dysplasia and heterotopic grey matter are the most commonly confused lesions with schizencephaly in the differential diagnosis. In FLAIR sequences, unlike schizencephaly, heterotopic grey matter islands are seen as hyperintense. In the FLAIR sequence, the white grey matter separation is clear as in the T1 sequence. Focal thickness increases of the cortex are well evaluated in FLAIR sequence $(11,12)$.

In a study on normal diffusion values, ADC values in grey matter were $0.8-1.1 \times 10^{3} \mathrm{~mm}^{2} / \mathrm{s}$ and those in white matter were $0.6-0.9 \times 10^{3} \mathrm{~mm}^{2} / \mathrm{s}$ (13). Grey matter values in the cortex in the $\mathrm{SCH}$ cleft in the ADC measurement made from grey matter were normally close to the lower limit of the specified limit. As is known, the main cause of $\mathrm{SCH}$ is abnormal migration. It is known that the cortical covering is abnormal.

In the SWI sequence, phase images are first formed. Filtered phase images are obtained from these phase images using high and low pass filters. Phase images are used to increase the contrast in the original magnified images. Magnified SWI images are obtained by reproducing phase images and combining them with the original magnified images. Afterwards, images are obtained using the minimum intensity projection (MIP $\geq 4$ image). SWI is a technique commonly used to detect bleeding and calcifications. Therefore, an abnormal appearance is not expected in schizencephaly. How- 
ever, it is helpful in the differential diagnosis (14).

Contrast-enhanced examinations are helpful in showing meningioma and accompanying vascular malformation. The piamater, which extends from the ependymal layer in schizencephaly, terminates in a wide cleft in closed lip schizencephaly. Contrast-enhanced sequences are useful for piamater evaluation.

\section{CONCLUSIONS}

Schizencephaly is one of the rare neuronal migration pathologies. It can be asymptomatic in cases when it is closed-lip and mild, as in our case. When detected, the presence of polymicrogyria, cortex thickness, and extension to the lateral ventricle should be evaluated. Differential diagnosis should be made especially with heterotopias and focal cortical dysplasia. Every MRI sequence have different importance according to the structure we want to see. T2 weighted images are the best to show polymicrogyria. White matter grey matter separation can be observed with FLAIR and T1 sequences. The benefit of diffusion-weighted images in schizencephaly is unclear. However, ADC values are close to the lowest limit of the normal values in deteriorated grey matter. SWI sequence has no place in schizencephaly except to the exclusion of vascular malformations and haemorrhage.

\section{Acknowledgement}

Endless thanks to Dr. Marwa Mouline Doğan for her assistance.

Conflict of interest: none declared Financial support: none declared

\section{REFERENCES}

1. Siti BC, Zulkifli MM, Mohd Yusoff SS, Muhamad R, Ahmad TM. A Rare Case of an Infant with Left Hemiparesis: A Case Report of Bilateral Open-lip Schizencephaly. Malays Fam Physician. 2020 Nov 10;15(3):90-94.

2. Hung PC, Wang HS, Chou ML, Lin KL, Hsieh MY, Chou IJ, Wong AM. Schizencephaly in children: A single medical center retrospective study. Pediatr Neonatol. 2018 Dec;59(6):573-580.

3. Vézina G. Congenital malformations of the brain: prenatal and postnatal imaging. Semin Roentgenol. 2004 Apr;39(2):165-181.

4. Gonzalez JC, Singhapakdi K, Martino AM, Rimawi BH, Bhat R. Unilateral Open-lip Schizencephaly with Tonsillar Herniation in a Preterm Infant. J Pediatr Neurosci. 2019 Oct-Dec;14(4):225-227.

5. Veerapaneni P, Veerapaneni KD, Yadala S. Schizencephaly. 2020 Aug 10. In: StatPearls [Internet]. Treasure Island (FL): StatPearls Publishing; 2020 Jan.

6. Denis D, Chateil JF, Brun M, Brissaud O, Lacombe D, Fontan D, Flurin V, Pedespan J. Schizencephaly: clinical and imaging features in 30 infantile cases. Brain Dev. 2000 Dec;22(8):475-483.

7. Griffiths PD. Schizencephaly revisited. Neuroradiology. 2018;60(9):945-960.

8. Barkovich AJ, Norman D. MR imaging of schizencephaly. AJR Am J Roentgenol. 1988;150(6):1391-1396.

9. Fadakar K, Dadkhahfar S, Esmaeili A, Keyhanidoust Z. A case of schizencephaly and septo-optic dysplasia presenting with anterior encephalocele. Iran J Child Neurol. 2012;6(4):47-50.

10. Arboix A, González-Peris S, Grivé E, et al. Cortical laminar necrosis related to migrainous cerebral infarction. World J Clin Cases. 2013;1(8):256-9.

11. Kabat J, KróL P. Focal cortical dysplasia - review. Pol J Radiol. 2012;77(2):35-43.

12. Barkovich AJ, Kuzniecky RI, Jackson GD, et al. A developmental and genetic classification for malformations of cortical development. Neurology. 2005;65(12):1873-87.

13. Helenius J, Soinne L, Perkiö J, Salonen O, Kangasmäki A, Kaste M, Carano RA, Aronen HJ, Tatlisumak T. Diffusion-weighted MR imaging in normal human brains in various age groups. AJNR Am J Neuroradiol. 2002 Feb;23(2):194-9.

14. Barbosa JH, Santos AC, Salmon CE. Susceptibility weighted imaging: differentiating between calcification and hemosiderin. Radiol Bras. 2015 Mar-Apr;48(2):93-100. 